\title{
THE NOVEMBER MEETING IN LEXINGTON
}

The five hundred thirtieth meeting of the American Mathematical Society was held at the University of Kentucky in Lexington, Kentucky on Friday and Saturday, November 30-December 1. About 95 persons registered, including 60 members of the Society.

By invitation of the Committee to Select Hour Speakers for Southeastern Sectional Meetings, there were two hour addresses. Professor L. M. Milne-Thomson, of the Royal Naval College of Greenwich (visiting Professor at Brown University) spoke Friday evening on Some hydrodynamical methods, and Professor O. G. Harrold, of the University of Tennessee spoke Saturday morning on Locally tame curves and surfaces in 3-manifolds. Professors Tomlinson Fort and H. C. Griffith presided at these sessions.

There were four sessions for contributed papers, Professors R. D. Anderson, V. F. Cowling, M. L. Curtis and Frank Levin presiding.

Abstracts of the papers presented follow. Those having the letter " $t$ " after their numbers were read by title. Where a paper has more than one author, that author whose name is followed by "(p)" presented it. Mr. Hunter was introduced by Professor R. J. Koch.

\section{Algebra and Theory of Numbers}

123t. Eckford Cohen: Congruence representations in algebraic number fields II. Simultaneous linear and quadratic congruences.

Let $P$ be an odd prime ideal in a finite extension $F$ of the rational field. In this paper the author determines the number of simultaneous solutions $N_{s}(m, n)$ of the pair of congruences $m \equiv \alpha_{1} x_{1}^{2}+\cdots+\alpha_{8} x_{g}^{2}, n \equiv \beta_{1} x_{1}+\cdots+\beta_{s} x_{s}\left(\bmod P^{\lambda}\right)$ where $m$ and $n$ are arbitrary integers of $F$ and the $\alpha_{i}, \beta_{i}$ are integers of $F$ prime to $P$. The case $\lambda=1$ was treated earlier, using the terminology of Galois fields (Bull. Amer. Math. Soc. Abstract 62-1-3). The method of the paper is based on the elementary theory of Cauchy-Gauss sums in $F$. Explicit results for $N_{s}(m, n)$ are deduced, and it is shown that the least value of $s$ such that $N_{s}(m, n)>0$ for all odd prime-power ideals $P^{\lambda}$, for all $m, n$, and all $\alpha_{i}, \beta_{i}$ prime to $P$ is the value $s=5$. This contrasts with the minimal value $s=4$ under the restriction $\lambda=1$. (Received September 24, 1956.)

\section{W. E. Deskins: $A$ note on group representations.}

A subgroup $H$ of a finite group $G$ is defined to have property $I$ if each irreducible representation module of $G$ (over the field $F$ ) remains irreducible when considered as a representation module for $H$. The following results are obtained: THEOREM 1. Let $H$ have property $I$ and let $g$ denote the order of $G$. Then $H$ is a normal subgroup and contains the commutator subgroup of $G$ provided (1) the characteristic of $F$ is relatively prime to $g$; (2) $g=q p^{a},(p, q)=1, p$ the characteristic of $F$, and the order of $H$ is $p^{a}$; or (3) the radical of the group-ring of $G$ is generated by the radical of the group-ring of $H$. Examples which demonstrate that the theorem is not necessarily true if (1), (2) or 
(3) is not satisfied exist. Theorem 2. If $H$ has property I and condition (1) is satisfied, then not only is $H$ normal but each conjugate class of $H$ is also a conjugate class in $G$. Examples which illustrate that the result may be false if (1) is not satisfied exist. The question, Given a group $H$, does there exist a nontrivial Abelian extension $G$ such that $H$ possesses property I? is answered affirmatively. (Received October $3,1956$.

\section{D. W. Wall: $A$ note on generalized uniserial algebras.}

Let $A$ be an algebra over an algebraically closed field $K$. Let $L$ be a primitive left ideal dual to some primitive right ideal. Thus, $L$ has a unique minimal subideal $L_{1}$. If, in addition, it is assumed that $L$ has only one subideal containing $L_{1}$ as a maximal subideal and that $L_{1}$ and $L_{2} / L_{1}$ are $A$-isomorphic as left $A$-spaces, then it can be shown that $L$ has only one composition series and all of the constituents $L_{i} / L_{i-1}$ of $L$ are $A$-isomorphic to $L_{1}$. Examples are given to show that if either of the two hypotheses is dropped then neither of the two conclusions need hold. With the aid of this and additional lemmas, it is possible to extend an earlier result: If for every two-sided ideal $Z, A / Z$ is a $Q F-2$ algebra then $A$ is a generalized uniserial algebra (Bull. Amer. Math. Soc. Abstract 62-2-181). The $Q F-2$ algebras form a subclass of the $Q F-3$ algebras which are those having unique minimal faithful representations (Thrall, Trans. Amer. Math. Soc. vol. 64 (1948) pp. 173-183). The theorem is still true if the hypothesis that every $A / Z$ be a $Q F-2$ algebra is replaced by weaker hypotheses requiring only that every $A / Z$ belong to more general classes of $Q F-3$ algebras. (Received October 4, 1956.)

\section{6t. F. B. Wright: Ideals in a polyadic algebra.}

Halmos has shown that the Gödel completeness theorem for the first-order functional calculus is equivalent to the (polyadic) semisimplicity of a polyadic Boolean algebra. It is shown in this note that polyadic semisimplicity is a corollary of the following two results. (1) Let $A$ be a polyadic $I$-algebra, with quantifier function $E$, and let $B$ be the range of the quantifier $E(I)$. Then there exists a one-to-one correspondence between the ideals of $B$ and the polyadic ideals of $A$. (2) Every ideal of $A$ contains a polyadic ideal of $A$. (Received September 28, 1956.)

\section{A. M. Yaqub: $A$ note on the commutativity of a certain class} of rings.

Let $p$ be prime. A ring $R$ with unit is called $p$-like if it is of characteristic $p$ and if, for every $x, y \in R,(x y)^{p}-x^{p}-x^{p} y+x y=0$. $p$-like rings essentially generalize the Boolean rings of Stone (Trans. Amer. Math. Soc. vol. 40 (1936) pp. 37-111), the prings of McCoy and Montgomery (Duke Math. J. vol. 3 (1937) pp. 455-459), and the Boolean-like rings of Foster (Trans. Amer. Math. Soc. vol. 59 (1946) pp. 166-187). It is shown that $p$-like rings are commutative. The proof is elementary and follows after some properties of such rings are established. An elementary number-theoretic result is also used in the proof. (Received September 24, 1956.)

\section{ANALYsis}

128. W. K. Ergen, H. J. Lipkin and J. A. Nohel (p): Applications of Liapounov's second method in reactor dynamics.

Liapounov's Second Method, which establishes sufficient conditions for stability of solutions of nonlinear differential equations, is used to obtain criteria which guarantee stability of solutions of reactor equations. Conditions for stability are obtained 
both for homogeneous and heterogeneous reactors, where in the latter case heat is generated in each of the $n$ reactor media. The important special cases of two and three media as well as the general case of $n$ media are studied. Aspects of nonlinear reactor dynamics of the type studied here were considered previously from the physical point of view by W. K. Ergen and A. M. Weinberg, Some aspects of non-linear reactor dynamics, Physica XX (1954), and H. J. Lipkin, A study of the nonlinear kinetics of the Chatillon reactor, J. Nuc. En. vol. 1 (1955). The heterogeneous reactor considered by Lipkin had heat generated in only one medium. The present paper sets these earlier investigations on a firm mathematical basis and generalizes Lipkin's work on the Chatillon Reactor. (Received October 1, 1956.)

129. Tomlinson Fort: The five-point difference equation with periodic coefficients.

In this paper the author considers the five-point difference equation in two independent variables over a finite domain of discrete points. The coefficients are periodic in one of the variables. The second variable is limited to a domain of extent equal to the period in the first variable. A number of theorems are proved relative to constants, $\rho_{i}$, which are roots of an equation called the characteristic equation. In particular, theorems are proved relative to the form of solutions which constitute a certain fundamental system, that is, solutions in terms of which all solutions can be linearly expressed. (Received October 4, 1956.)

130. R. M. McLeod (p), J. J. Gergen, and F. G. Dressel: Uniqueness of mapping pairs for elliptic equations.

Let $D^{*}$ be a simple, closed Jordan curve with a representation $z=\phi(t), a \leqq t \leqq b$, such that $\phi^{\prime}(t)$ exists for $a \leqq t \leqq b, \phi^{\prime}(a)=\phi^{\prime}(b), \phi^{\prime}(t) \neq 0$ for all $t$ in $[a, b]$, and $\left|\phi^{\prime}\left(t_{1}\right)-\phi^{\prime}\left(t_{2}\right)\right| \leqq N\left|t_{1}-t_{2}\right|^{\nu}$ for $t_{1}, t_{2} \in[a, b]$, where $0<N, 0<\nu \leqq 1$. Let $D$ be the interior of $D^{*}$ and set $D^{c}=D \cup D^{*}$. Let $\alpha, \beta, \gamma, \delta$ be real-valued functions of class $C^{0}$ on $D^{c}$ and $C^{\prime}$ on $D$ with bounded derivatives on $D$. Further suppose $\alpha>0,4 \alpha \delta$ $-(\beta+\gamma)^{2}>0$ on $D^{c}$. It is shown that, if $w=F(z), F=u+i v$, is a homeomorphism of $D^{c}$ onto a plane set with $F \in C^{\prime}$ on $D$ and $\alpha u_{x}+\beta u_{y}=v_{y}, \gamma u_{x}+\delta u_{y}=-v_{x}$ on $D$, then $F$ is determined uniquely by its values at three distinct points of $D^{*}$. The case $\alpha=\delta$, $\beta=\gamma=0$, and the domain $D$ a circular disk was treated earlier by Gergen and Dressel (Duke Math. J. vol. 19 (1952) pp. 435-444). (Received October 4, 1956.)

131. E. P. Miles, Jr. (p) and Ernest Williams: Properties of functions associated with the Cauchy problem for the damped wave equation. Preliminary report.

In a recent abstract (62-2-211) solutions of the form $\sum_{s-0}^{i} \nabla^{2 s} F \cdot u_{s}$ for the damped wave equation with polyharmonic initial values $F$ were obtained. The functions $\left\{u_{s}\right\}$ are shown to satisfy the recursion formulas: $u_{s}=1 / 2 s(2 s-2) k^{2} \cdot\left[(2 s-2)(2 s-3) u_{s-1}\right.$ $\left.+t^{2} u_{t-2}\right]$ and $u_{s}=-t / 2 s \int_{0}^{t} u_{t-1} d t$. The polynomials $P^{s}(k t)$, which are the coefficients of $e^{-k t}$ in the expansions of $u_{a}$, are shown to be solutions of a fourth order ordinary differential equation. (Received October 4, 1956.)

\section{2t. B. J. Pettis: Comment on a theorem of Jerison.}

Using a net theorem previously established by the author it is possible to rephrase and extend a result by Jerison (Proc. Amer. Math. Soc. vol. 5 (1954)) concerning the 
comparison of cluster points for a net in a linear topological space and for the net of closed convex covers. (Received October 8, 1956.)

\section{Geometry}

\section{V. G. Grove: On closed convex surfaces.}

The purpose of this paper is to prove the theorem: let $S$ and $\bar{S}$ be two closed orientable convex surfaces of class $C^{\prime \prime \prime}$ imbedded in a Euclidean space of three dimensions, and possessing no parabolic points. Let $h$ be a differentiable homeomorphism of $S$ into $\bar{S}$ such that $h I I=\overline{I I}, I I$ and $\overline{I I}$ being the second fundamental forms of $S$ and $\bar{S}$ respectively, such that the Gaussian curvatures $K$ and $\bar{K}$ of $S$ and $\bar{S}$ are equal at corresponding points, and such that the orientations of $S$ and $\bar{S}$ are preserved. Then $h$ is a rigid motion. (Received October $3,1956$. )

\section{TOPOLOGY}

134. R. D. Anderson: On extending homeomorphisms and a form of homogeneity.

Let $X$ be a compact metric continuum and let $S$ be a collection of closed subsets of $X$. Then $X$ is said to be homogeneous with respect to $S$ if for any homeomorphism $g$ of an element $s$ of $S$ onto an element $s^{\prime}$ of $S$ there exists a homeomorphism $f$ of $X$ onto itself, with for $x \in s, f(x)=g(x)$. The principal result of this paper is the theorem that the universal curve $M$ is homogeneous with respect to the set $S$ of all closed subsets of $M$ which (1) do not contain open subsets of $M$ and (2) do not separate connected open subsets of $M$. It may be noted that (a) $S$ contains all countable closed subsets of $M$ and (b) $S$ is maximal in the sense that $M$ cannot be homogeneous with respect to any collection $S^{\prime}$ of closed subsets of $M$ with $S^{\prime}$ containing $S$ as a proper subset. That (b) is so follows from the fact that $S$ contains a homeomorphic image of $M$ and hence homeomorphic images of all closed subsets of $M$. (Received October 4, 1956.)

135. C. E. Capel (p) and W. L. Strother: Counterexample to a theorem of Hamilton.

In a paper of O. H. Hamilton [Duke Math. J. vol. 14 (1947) pp. 689-693] appears the statement that if $T$ is a continuous multi-valued transformation of an $n$-cell into itself such that the image of each point is an $n-1$ sphere then $T$ has a fixed point. A counterexample to this is given. (Received September 20, 1956.)

\section{M. L. Curtis: An imbedding theorem.}

R. H. Bing has defined a decomposition of $E^{3}$ into points and tame arcs such that the decomposition space $M^{3}$ is topologically different from $E^{3}$ (see page 45 of the Set Theoretic Topology Institute Summary, Madison, Wisconsin, 1955). As an application of the imbedding theorem of this paper we show that this space $M^{3}$ can be imbedded in $E^{4}$. (Received September 27, 1956.)

137. M. K. Fort, Jr.: A note concerning a decomposition space defined by Bing.

Recently (see pp. 44-47, Summary of lectures and seminars, Summer Institute on Set Theoretic Topology, Madison, Wisconsin, 1955), R. H. Bing has defined a decom- 
position of $E^{3}$ into points and tame arcs in such a way that the decomposition space is not homeomorphic to $E^{8}$. In this note, it is shown that Bing's construction can be carried out in such a way that the tame arcs are actually broken line segments, each of which has exactly two breaks. The breaks all occur on two parallel planes in $E^{3}$; and if $\theta$ is a positive number, it is possible to require that no one of the broken line segments contain an angle that differs from a straight angle by more than $\theta$. (Received September 28, 1956.)

\section{8t. Morikuni Goto and Naoki Kimura: Semigroups of endomor- phisms of a locally compact group.}

The structures of the semigroups of endomorphisms of a locally compact group will be studied in detail. (Received October 1, 1956.)

\section{H. C. Griffith: A characterization of tame surfaces in three} space.

Let $C$ be a 2-cell in 3-space and let $J$ be the set of all arcs which either lie in $\partial C$ or meet $\partial C$ only in their endpoints. Each $T_{i}$ in $J$ determines two closed sets $M_{i}$ and $N_{i}$ such that $M_{i} \cap N_{i}=T_{i}$ and $M_{i} \cup N_{i}=C$, and a metric $\rho$ on $J$ is defined by $\rho\left(T_{1}, T_{2}\right)=\min \left[\sigma\left(M_{1}, M_{2}\right)+\sigma\left(N_{1}, N_{2}\right), \sigma\left(M_{1}, N_{2}\right)+\sigma\left(M_{2}, N_{1}\right)\right]$, where $\sigma$ is the Hausdorff metric. Let $D(T, \epsilon)$ denote the set of all disks such that $1^{\circ} \partial D \cap C=\square$, $2^{\circ} D \cap C \in J, 3^{\circ} \rho(D \cap C, T)<\epsilon$, and $4^{\circ}$ if $C \backslash D$ has two components, then $D$ separates them in every sufficiently small neighborhood of $C$. If $D(T, \epsilon) \neq \square$ for all $T \in J$ and $\epsilon>0$, then $C$ is said to have the disk property. If to each pair of disjoint open subsets of $\partial C$ there correspond a pair of disjoint topological rays, one emanating from each of the two sets, which are otherwise disjoint from $C$ and have the property that to each $\epsilon>0$ there is a 2-sphere in $S(C, \epsilon)$ enclosing $C$ and meeting each of the rays in a single point, then $C$ has the strong enclosure property. Some cells are such that if $D_{1}$ and $D_{2}$ are disjoint disks in $\bigcup D(T, \epsilon)$ and $T^{\prime} \in J$ meets each in a single point then to each $\epsilon>0$ there is a $D$ in $D\left(T^{\prime}, \epsilon\right)$ such that $D \cap D_{i} \cap C$ is a point, $i=1,2$. It is shown in this paper that a cell with a disk property and the strong enclosure property is tame if and only if it meets this condition. (Received October 2, 1956.)

\section{J. G. Horne, Jr.: o-ideals and the ideal structure of certain rings.}

The role played by Milgram's $o$-ideals in studying the ring of continuous functions $C(X)$ on a topological space $X$ has led to a study of this notion in an abstract commutative ring $R$ with identity. (Bull. Amer. Math. Soc. Abstracts 62-1-116 and 62-2-276). If $R$ satisfies the condition $\left({ }^{*}\right)$ : every maximal (equivalently: every prime) ring ideal contains a maximal $o$-ideal, then the collection $\operatorname{Tr}(R)$ of maximal $o$-ideals in $R$ forms a compact Hausdorff space in the dual Stone Topology. In such rings, the class of maximal o-ideals coincides with the class of prime-like o-ideals (in Abstract 62-1-116 these were called $p$-ideals). In order that $R$ satisfy condition $(*)$ it is sufficient that $R$ be an r-ring: $e f=f$ implies there are $h, e \in R$ such that (i) $e^{\prime} f=f$ and (ii) (he) $e^{\prime}$ $=e^{\prime}$. In general, when $\mathscr{T}(R)$ is compact and $\cap\{M: M \in \mathfrak{T}(R)\}$ is zero, then for every ring ideal $J, J=\bigcap\{M+J: M \in \mathscr{N}(R)\}$. This result implies in particular that every $o$-ideal is the intersection of all maximal o-ideals containing it, as well as a theorem on "belonging locally to an ideal" in commutative Banach algebra theory. (Received October 4, 1956.)

\section{R. P. Hunter: Concerning mobs.}

If $S$ is a decomposable clan, then for any $n$ there is a collection of $n$ subcontinua 
whose finished sum is $S$. This generalizes a result of Koch and Wallace (Duke Math. J. vol. 21 (1954) pp. 681-686). If the clan $S$ is nearly 2-homogeneous over $x$ and $y$ such that $y$ is in $T(x)$ then $S$ is a group. If $S$ is indecomposable with proper kernel then one and only one composant $Q$, is an ideal; $Q$ is an indecomposable connexe containing $S^{2} ; S$ is irreducible between a subset $A$ and $A^{n}$ if and only if $A$ does not meet $Q ; S$ contains a dense ideal $B$ different from $Q$ which is an indecomposable connexe and such that $f(B)$ is widely connected where $f$ is the canonical map mod $S^{2}$. (Swingle Proc. vol. 2 (1951) pp. 178-185). If the mob $S$ is the finished sum of two indecomposable continua and has proper kernel then $S$ is not irreducible between two idempotents. (Received October 4, 1956.)

\section{John Jewett: Differentiable approximations to interior func- tions.}

The following is proved: THEOREM. Let $D$ be a domain in the plane and let $f: D^{-} \rightarrow R^{1}$ be a real valued function which is continuous on $D^{-}$and interior on $D$ into $R^{1}$. Let $n$ be a positive integer. Then for each positive number $\epsilon$ there exists a function $g: D^{-} \rightarrow R^{1}$ such that (1) $|f(z)-g(z)|<\epsilon$ for all points $z$ of $D^{-}$, (2) $g$ is continuous on $D^{-}$and interior on $D$ into $R^{1}$, (3) $g$ is $n$ times continuously partially differentiable, and (4) $g$ agrees with $f$ on the boundary of $D$. The proof makes use of an approximation theorem proved by the author (Duke Math. J. vol. 23 (1956) pp. 111-124) for light interior mappings together with a deformation process. A similar theorem is proved for a class of monotone mappings of plane domains. (Received October 1, 1956.)

\section{R. J. Koch (p) and A. D. Wallace: Admissibility of semigroup structures on continua.}

A class of compact connected spaces is exhibited which do not admit the structure of a mob equal to its square except in the trivial ways $x y=x($ all $x, y)$ or $x y=y$ (all $x, y)$. Included in this class are the unit circumference (when it is not a group) and the figure eight. The arguments make use of the following lemma: a compact connected mob $S$ satisfies $S^{2}=S$ if and only if each dense left ideal containing $K$ (the minimal ideal) is connected. Further, we investigate the structure of a compact connected mob $S$ equal to its square and irreducible between $K$ and $A$ where $A$ is closed and misses $K$. Such a mob has a unit, a group kernel, and each continuum meeting $K$ and $S-K$ is a principal ideal. Further if $\mathrm{cd}(S / K)=1$ then $S$ is commutative. (Here cd represents the cohomology dimension). (Received October 4, 1956.)

144. J. D. McKnight, Jr. (p), R. W. Bagley and E. H. Connell: Compactness conditions I. Pseudocompact spaces.

Let $C(X)$ denote the set of real-valued continuous functions on a space $X$, and $C^{*}(X)$ the bounded elements of $C(X)$. A space $X$ has the property that every sequence of $C(X)$ that converges uniformly at every point of $X$ converges uniformly on $X$ if and only if $C(X)=C^{*}(X)$. If $X$ has this property it is called pseudocompact [cf. Hewitt, Trans. Amer. Math. Soc. vol. 64 (1948) p. 67]. Further, $C$ is pseudocompact if and only if every sequence in $C(X)$ that converges locally uniformly on $X$ converges uniformly on $X$. These characterizations of pseudocompactness lead heuristically to an interesting topological characterization for completely regular spaces: $X$ is lightly compact if every locally finite collection of open sets of $X$ is finite. There are, however, pseudocompact regular spaces that are not lightly compact (light compactness always implies pseudocompactness). The equivalence of pseudocompactness 
and light compactness for completely regular spaces furnishes an immediate proof that pseudocompactness and countable compactness are equivalent for normal $T_{1}$ spaces and makes it easy to find an example of a completely regular locally compact and pseudocompact $T_{1}$ space that is not countably compact. Moreover, compactness in completely regular spaces is characterized as pseudocompactness plus paracompactness. (Received October 3, 1956.)

145. J. D. McKnight, Jr., R. W. Bagley (p) and E. H. Connell: Compactness conditions II : Lightly compact spaces.

Light compactness is exactly the condition needed in addition to paracompactness to yield compactness. Moreover, a space is countably compact if and only if it is lightly compact and countably paracompact. The following five statements are equivalent: (i) $X$ is lightly compact; (ii) No infinite sequence of pairwise disjoint open sets of $X$ is locally finite; (iii) Every closed neighborhood properly contained in $X$ is lightly compact; (iv) Every countable open covering of $X$ contains a finite subcollection whose closures form a covering; (v) If $\left\{x_{n}\right\} \subset X$ is countably infinite and $\left\{U_{n}\right\}$ is a countable collection of open sets covering $X$, then there is some member of $\left\{\bar{U}_{n}\right\}$ containing infinitely many points of $\left\{x_{n}\right\}$. In a lightly compact space, the Lindelöf property is equivalent to property $\left({ }^{*}\right)$ : Every open covering has a $\sigma$-locally finite open refinement. A regular space is compact if and only if it is lightly compact and has property (*), from which it follows that a completely regular space is compact if and only if it is pseudocompact and has property (*). In a metric space light compactness (pseudocompactness) and compactness are equivalent. In a $T_{1}$ space compactness is equivalent to light compactness plus property $\left({ }^{*}\right)$ plus the property that every point has a neighborhood whose boundary is countably compact. (Received October 3,1956 .)

\section{6t. A. D. Wallace: Remarks on the center of a lattice.}

A lattice is a Hausdorff space together with a pair of continuous lattice operations. Theorem 1 . The center of a compact lattice is totally disconnected. Theorem 2 . If a compact connected lattice is topologically immersed in Euclidean space then its center is contained in its boundary. Thus, far from being centrally located, the central elements are peripheral. If the lattice above is modular then any complemented element is peripheral. (Received September 14, 1956.) 